\title{
Finanzierung überprüfen
}

\section{Lohnt sich ein Bankenwechsel?}

\begin{abstract}
Beim Immobilienkauf oder bei anderen größeren Investitionen wird die einmal gewählte Finanzierung oft nicht hinterfragt. Dabei lohnt sich ein Finanzierungsvergleich häufig: Wer verschiedene Angebote einholt, kann nicht nur bei der neuen Anschlussfinanzierung viel Geld sparen, sondern unter Umständen auch die Rückzahlungsdauer erheblich verkürzen. Die Interhyp AG hat fünf Tipps zusammengestellt, worauf besonders zu achten ist.
\end{abstract}

\section{Tipp 1: Angebote vergleichen und Bankwechsel in Erwägung ziehen} Besonders für Immobilienbesitzer, deren Anschlussfinanzierung bald bevorsteht, sind die Möglichkeiten zur Zinsoptimierung aktuell sehr groß: Hausund Wohnungskäufer haben bei einem Darlehensabschluss vor rund zehn Jahren einen Zinssatz von über sechs Prozent effektiv gezahlt, die heutigen Bestsätze für zehnjährige Baudarlehen hingegen liegen bei derzeit rund vier Pro-

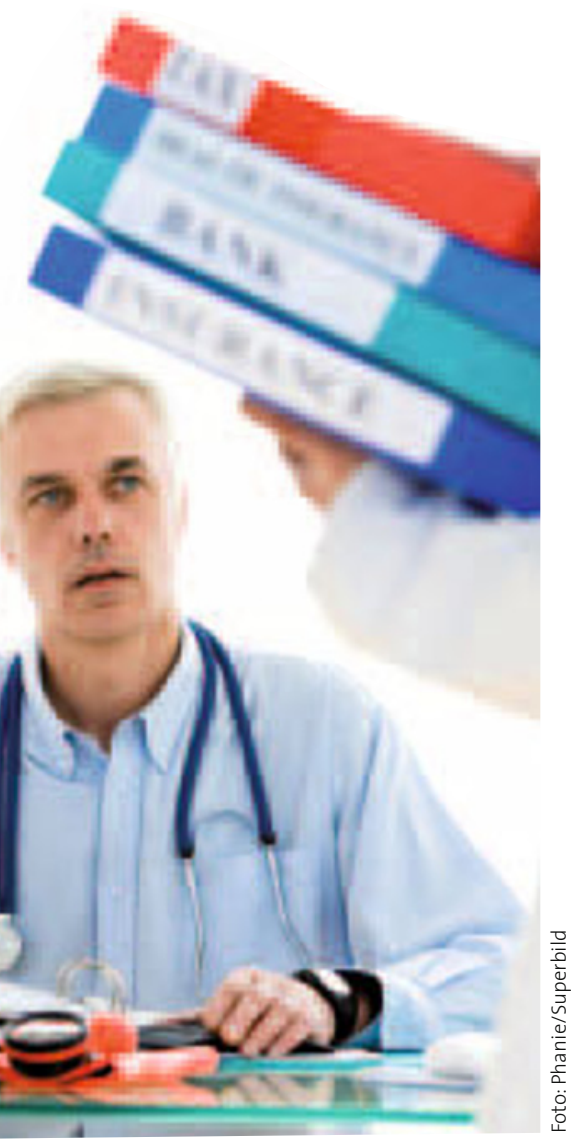

Angebote vergleichen lohnt sich. zent. Spätestens sechs Monate vor dem Ende der bisherigen Zinsbindung sollte man seine Vertragsbedingungen prüfen und aktuelle Vergleichsangebote einholen, am besten bei anbieterunabhängigen Vermittlern, so der Ratschlag der Interhyp AG. Scheu vor einem Anbieterwechsel müsse man dabei nicht haben. Der Wechsel zu einer anderen Bank sei mühelos möglich und koste nicht viel: Für eine Grundschuldabtretung, die die Finanzinstitute meist unter sich regeln, ist bei einer Grundschuld von 100000 Euro mit Gebühren von 200 bis 400 Euro zu rechnen, erklärt die Interhyp AG.

\section{Tipp 2: Erreichte Tilgung beibehalten}

Bei einem Ausgangs-Zinssatz von 6\% hat man durch die Mechanik des Annuitätendarlehens nach zehn Jahren seine anfängliche Tilgung von 1\% auf gut $2 \%$ erhöht. Keinesfalls sollte man diese Tilgungshöhe bei den jetzt niedrigeren Zinsen wieder auf den anfänglichen Satz von 1\% reduzieren. Denn damit verlängert sich nur unnötig die Rückzahlung des Kredites.

\section{Tipp 3: Zinsvorteile für höhere Tilgung verwenden}

Immobilienbesitzer, die ihre Monatsrate in der Vergangenheit problemlos bewältigen konnten, sollten diesen Betrag beibehalten und die Zinsersparnis direkt für eine schnellere Tilgung aufwenden, so der weitere Ratschlag der zierung mit einprozentiger Anfangstilgung aufgenommen hat, kann heute Interhyp AG. Wer 1999 eine Baufinan- bei gleichbleibender Kreditrate z. B. $3,9 \%$ Tilgung vereinbaren und damit neun Jahre früher schuldenfrei sein.

\section{Tipp 4: Lange Zinsbindung bis zur Schuldenfreiheit wählen}

Die Kombination aus niedrigeren Zinsen und einem deutlich höheren Tilgungsspielraum ermöglicht Planungssicherheit und schnellere Schuldenfreiheit. „Benötigt man mit der entsprechenden Tilgung ohnehin nur 15 bis 20 Jahre bis zur vollständigen Rückzahlung, sollte man eine Zinsbindung wählen, die diesen Zeitraum vollständig deckt“, empfiehlt Robert Haselsteiner, Vorstand der Interhyp AG. Dafür sprechen nicht nur die für langfristige Laufzeiten derzeit günstigen Konditionen, sondern man vermeidet eine weitere Anschlussfinanzierung und das damit verbundene Risiko höherer Zinsen.

\section{Tipp 5: Eigenkapitaleinsatz prüfen}

Wer zum Beispiel auf dem Tagesgeldkonto Kapital verfügbar hat, sollte angesichts der derzeit wenig attraktiven Guthabenverzinsung prüfen, ob er dieses Vermögen zumindest teilweise für die Rückzahlung des Darlehens einsetzt. Denn das Zinsbindungsende ist der ideale Zeitpunkt, um durch eine Einmalzahlung die Kreditschuld ohne Zusatzkosten zu reduzieren. Positiver Nebeneffekt dabei: Über die verringerte Restschuld kann sich ein günstigeres Verhältnis von Objektwert zu Darlehenssumme ergeben. Das wiederum belohnen die Banken mit noch niedrigeren Zinskonditionen.

Ein Vergleich der Anschlussfinanzierung und Verhandlungen mit der Hausbank können sich also durchaus lohnen.

\footnotetext{
- Autor: Anke Thomas Quelle: Interhyp AG Pressemitteilung: „Anschlussfinanzierung optimieren - gewusst wie" vom 29.4.2009
} 